\title{
The Study of Wavelet Analysis in Line Selection of Single-Phase Earth Fault in Medium Voltage Distribution Network
}

\author{
Changzhi Lv ${ }^{1, a}$, Fei Peng ${ }^{2, b}$ \\ ${ }^{1,2}$ CEEA. Shandong University of Science and Technology, Qingdao, Shandong 266590,China

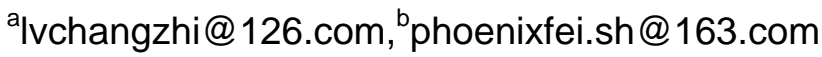

\begin{abstract}
Key words: fault line, wavelet criteria, transient current, detected
Abstract: On the basis of detection fault line research about abstraction transient fault information in ground system, the fault line could be selected because of its high frequency transient current. After we discussed and analyzed the wavelet criteria of the detected signal in theory, the significant amplitude of correlation analysis in the selected fault line can be proved. We put forward a fault line identifying principle based on the difference of magnitude with wavelet transform. At the same time, the model of the selection fault line in ground system can be simulated by MATLAB. In the paper, some obviously detected fault line features and the extraction zero-sequence currents of wavelet transform amplitude used in route selection have been tested in medium voltage distribution network with small current grounding system.
\end{abstract}

\section{Introduction}

Medium voltage distribution network means $6 \sim 10 \mathrm{KV}$ distribution network, In China Today, Neutral ungrounded method and Petersen-coil grounded way are widely used in medium voltage distribution network ${ }^{[1,2]}$. Single-phase ground fault account for more than $85 \%$ in distribution network fault. Although it can work temporarily for a period of time, if the fault is not removal timely, it will cause two phase short circuit more seriously ${ }^{[3]}$. So when single-phase earth fault occurred in medium voltage distribution network, it is vital significance to select fault line timely and accurately. When earth fault occurred in small current grounding system, three phase line voltage phase is still symmetry, earth fault current is much lower than load current. Especially in neutral earthing via arc extinguishing coil system, because of the earth fault current is much lower than load current., when earth faults occur, protection load and line without tripping immediately ,it also has no effect on the normal load, only relay protection equipment discrete sampling selectively ${ }^{[3]}$.

Fault line selection methods and corresponding devices which is known today are not ideal in the practical application, and also it has unavoidable defect. In this paper, wavelet transform is used to analyze zero-sequence current for relevant data, and then use the wavelet decomposition for transient component. The next step is to confirm characteristic scale and the amplitude of measured signal through wavelet transform coefficient and the certain detected frequency. Finally we confirm the feasibility of this method.

\section{Transient analysis of single-phase ground fault in small current grounding system}

The traditional criterion to select the fault line is judged by the steady-state component when single phase ground fault occurs in small current grounding system, lots of methods based on this criterion, such as comparing amplitude method, comparing phase method ,harmonic method and so on ${ }^{[4]}$. Although steady-state signal is continuous and stable and added to the characteristics that this signal can be tested by many methods ${ }^{[5]}$, its limitations determine that these methods can not select the fault line accurately when the system work is in the non-stable signal.

It is the equivalent circuit of neutral earthing via arc extinguishing coil system, shown in Figure 1: 


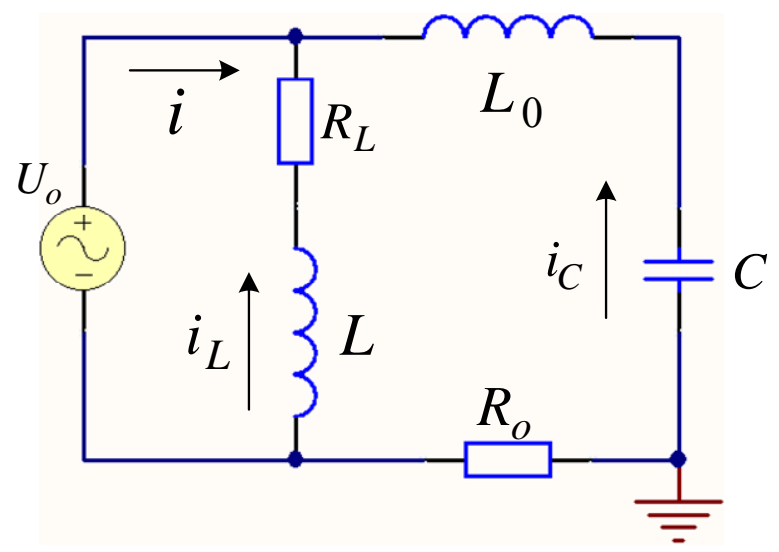

Figure 1. equivalent circuit of neutral earthing via arc extinguishing coil system The Eq.1 represent to the differential equation of transient capacitive current $\left(i_{C}\right)$ :

$$
R_{0} i_{c}+L_{0} \frac{d i_{c}}{d t}+\frac{1}{C} \int_{0}^{i} i_{c} d t=U_{\phi \mu} \sin (\omega t+\phi)
$$

If $R_{0}<2 \sqrt{\frac{L_{0}}{C}}$, the transient process of loop current oscillate and attenuate periodically .

If $R_{0}>2 \sqrt{\frac{L_{0}}{C}}$, the process is nonperiodic and settle out gradually.

The scope of the oscillation frequency is $300 \sim 3000 \mathrm{~Hz}$ in Medium voltage distribution network generally. The longer the lines, the oscillation frequency is lower, also the amplitude of oscillation component in transient capacitive current is lower, and it lasts for $0.5 \sim 1$ power frequency cycle ${ }^{[5]}$.

Eq. 2 refers to the equation of magnetic flux:

$$
\psi_{L}=\psi_{\text {st }}\left[\cos (\varphi+\xi) e^{\sqrt{\frac{t}{\tau L}}}-\cos (\omega t+\varphi+\xi)\right]
$$

In Eq. $2, \psi_{s t}=\frac{U_{\phi m}}{\omega W}$ represents the magnetic flux at steady state, $\xi=\operatorname{tg}^{-1} \frac{r_{L}}{\omega L}$ represents the impedance of arc-suppression coil, $t$ refers to the time constant in inductive loop. Because of $R_{L}<<\omega L$, we can get $Z \approx \omega L, \xi=0$. As it is known that $\psi_{L}=\psi_{o s}+\psi_{\text {st }}$, so Eq.2 can be simplified into Eq.3:

$$
\psi_{L}=\psi_{s t}\left[\cos \varphi e^{-\frac{t}{\tau L}}-\cos (\omega t+\varphi)\right]
$$

As it is known the equations $i_{L}=i_{L . d c}+i_{L . s t}$ and $I_{L m}=\frac{U_{\phi m}}{\omega L}$, the method to get the transient inductance current is as shown as Eq.4:

$$
i_{L}=i_{L m}\left[\cos \varphi e^{-\frac{t}{\tau L}}-\cos (\omega t+\varphi)\right]
$$

Eq.5 represents the expression to obtain transient grounding current :

$$
\begin{aligned}
i_{d}= & i_{C}+i_{L} \\
= & \left(I_{C m}-I_{L m}\right) \cos (\omega t+\varphi) \\
& +I_{C m}\left(\frac{\omega_{f}}{\omega} \sin \varphi \sin \omega t-\cos \varphi \cos \omega_{f} t\right) \\
& +I_{L m} \cos \varphi e^{-\frac{t}{\tau L}}
\end{aligned}
$$

The left side of Eq.5 denotes the steady state component, $\left(I_{C m}-I_{L m}\right) \cos (\omega t+\varphi)$ represents the D-value between the steady-state capacitance current and the steady-state inductance current, $I_{C m}\left(\frac{\omega_{f}}{\omega} \sin \varphi \sin \omega t-\cos \varphi \cos \omega_{f} t\right)+I_{L m} \cos \varphi e^{-\frac{t}{\tau L}}$ means the sum of transient free oscillation 
component in capacitance current and transient direct-current component in inductance current. According to the analysis about transient component above, although the transient process last just a short time when single-phase earth fault occurs in distribution network, the transient component has enormous fault information, and its amplitude is much higher than steady-state component ${ }^{[6]}$. For these reasons, it's a much more reliable and accurate way compared to steady-state component. At present, transient component is used to select the fault line widely in medium voltage distribution network when single-phase ground fault occurs.

\section{Line selection method based on wavelet transform of difference amplitude}

\subsection{Line selection management}

Phase frequency spectrum of wavelet transform has a lot of useful information, when selecting faulty lines, high-frequent parts in certain frequency band are extracted. The certain frequency band range in overhead line is $300 \sim 1500 \mathrm{~Hz}$, the certain frequency band range in cable line is $1500 \sim 3000 \mathrm{~Hz}$, according to Shannon sampling theorem $f \geq 2 f$ to decide sampling frequency of zero-sequence current of each line is $6 \mathrm{kHz}$.

In this paper, extracting the amplitude information is the basis of the method of using wavelet transform to extract the fault information. Firstly, according to scale - amplitude curves we can get a signal of the scale of the value, then signal amplitude can be determined. The measured signal amplitude $A_{x}$ and wavelet transform coefficient of amplitude that entering the steady state $\left|W_{x}\right|$ and feature scale $a$ formed the formula $A_{x}=c\left|W_{x}\right| / \sqrt{a}$, in the formula $c=2.008414$. We can see the amplitudes $A_{x}$ of signals are in direct proportion to wavelet transform coefficient of amplitude $\left|W_{x}\right|$. The basic step: the signal $x(t)$ to do Morlet complex wavelet transform, we get $a-S(a)$ curve, Finding out the corresponding scale value $a_{n}$, then work out the wavelet transform coefficient change cycle in scale value $a_{n}$, so we can get the frequency of the measured signal, then we can use $f_{a}=f_{s} f_{c} / a$ to get signal frequency corresponding to the characteristics of the scale, and signal amplitude can be worked out. Due to the difference of zero sequence current coefficient in fault phase and non-fault-phase, the fault line selection is supported. We use the former difference to select the fault line.

\subsection{Detection criterions}

Zero-sequence current is easier to extract, and it possesses a number of fault information., Added to the phenomenon that the transient variation trend of zero-sequence current in healthy phase is similar to a certain frequency in wavelet packets, while this trend is opposite to the trend of zero-sequence current in fault phase. When the busbar fault occurs, the variation trend of each single loop line is basically the same ${ }^{[7]}$. For these above-mentioned characteristics, zero-sequence current is used as the character of the earth fault line in this paper.

In order to reflect the difference in amplitude between healthy phase and fault phase, it's important to select an appropriate kind of wavelet. Different wavelet have different applied range and applied area. SymN wavelet supporting scope is $2 \mathrm{~N}-1$, vanishing moment order is $\mathrm{N}$, and it possesses good regularity. symN wavelet have similar function in continuity, support length, filter length compared to $\mathrm{dbN}$ wavelet, while it has better symmetry what means it can reduce phase distortion to a certain extent in signal analysis and reconstruction. After a great deal of simulation analysis, sym 5 wavelet can extract transient component of zero-sequence current efficiently, so sym5 wavelet is the best option in this paper.

When single-phase ground fault occurred in distribution network, the zero-sequence current amplitude of fault-phase is bigger than the amplitude of healthy-phase, and the variation trend of transient component is opposite between healthy-phase and fault-phase, so the wavelet transform module of transient zero-sequence current is a practicable criterion to select the fault line. $W I_{m}(a, b)$ represent the $b$ th number wavelet-decomposed coefficient of the $m$ th number line and the $a$ th number scale, so the criterion can obtain by Eq. 6: 


$$
M(n, m)=a b s\left(W I_{n}(a, b)\right)-a b s\left(W I_{m}(a, b)\right)
$$

$n, m$ represent the number of different lines ,and $n \neq m$.

First, extract the wavelet transform coefficients by sym5 from different lines, and then calculate the D-value of each two wavelet transform coefficients from every two lines, which write as $M(n, m)$, and finally, the fault line can be selected through the symbol of D-value $(M(n, m))$. As for the zero-sequence current of fault phase, it is much bigger than it in healthy phase, so the coefficient amplitude $\left|W_{n}\right|$ ( $n$th line is fault line)extracted from fault phase is bigger than other coefficient of healthy phase. Consequently, its D-values with healthy phases are all big positive values. Likewise, if $n$th line is not the fault line, there must have one or more small negative value in these D-values $(M(n, m))$.If ground earth fault happened on the busbar, the zero-sequence current amplitude of each line are similar, so the coefficient amplitudes are almost equal; in other words, the D-value $(M(n, m))$ is close to 0. For these above-mentioned reasons, the fault line can be selected through the criterion that whether these $\mathrm{D}$-values of every single line contain a big positive value.

The fault line selection process is shown in Figure 2:

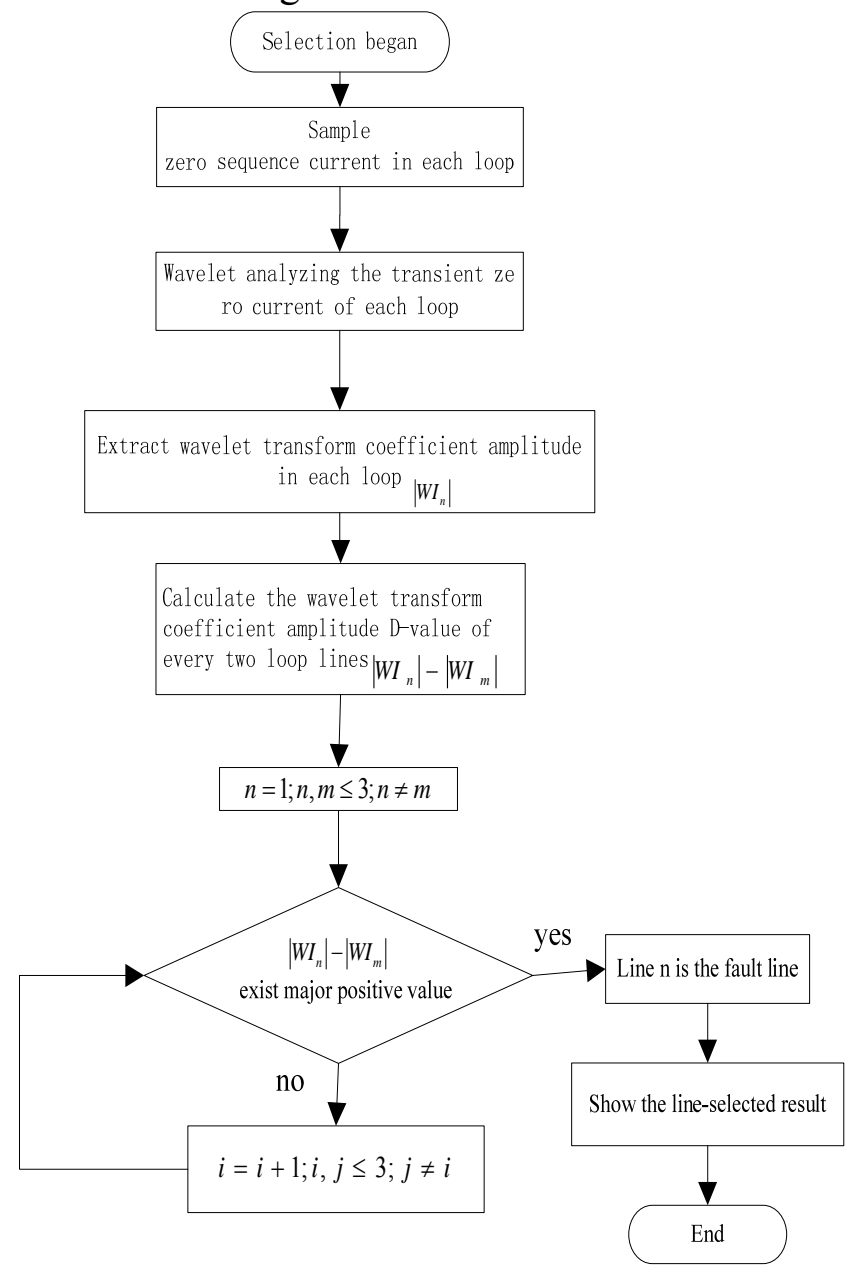

Figure 2. The fault line selection process

\section{Model}

The study software in this paper is Matlab and its Wavelet toolbox.

Power system use infinite capacity of three-phase power supply which is a ideal source. Positive sequence parameters: $R_{1}=0.45 \Omega / \mathrm{km}, L_{1}=0.9337 \mathrm{mH} / \mathrm{km}, C_{1}=0.07074 \mu \mathrm{F} / \mathrm{km} \quad$, zero-sequence parameters: $\quad R_{0}=0.74 \Omega / \mathrm{km} \quad, \quad L_{0}=4.1264 \mathrm{mH} / \mathrm{km}, C_{0}=0.0478 \mu \mathrm{F} / \mathrm{km}$. transformer reactance: $Z_{j}=0.5685+j 0.4033 \Omega$, arc suppression coil running way for excessive compensation. The compensation degree is $7.2 \%$.Length of line: the length of loop 1 is $25 \mathrm{~km}$, loop 2 is $8 \mathrm{~km}$,loop 3 is 
$25 \mathrm{~km}$. The change factors are based on ground resistance, the initial fault current angle(Based on phase A), fault location, line length, Take phase A in loop 1 as example to simulation testing. The result proves that this line selection method can be an accurate line selection in all kinds of failure modes.Line selection results in parts of simulations is showed in Table 1:

\begin{tabular}{cccccccccc}
\hline $\begin{array}{c}\text { Distance to } \\
\text { Fault } \\
(\mathrm{km})\end{array}$ & $\begin{array}{c}\text { transition } \\
\text { resistance } \\
(\Omega)\end{array}$ & $\begin{array}{c}\text { fault- incepti } \\
\text { on angle } \\
(\circ)\end{array}$ & \multicolumn{2}{c}{ loop 1 } & \multicolumn{2}{c}{ loop 2 } & \multicolumn{2}{c}{ loop 3 } & result \\
10 & 0.001 & 45 & + & + & - & - & - & + & loop 1 \\
10 & 10 & 45 & + & + & - & - & - & + & loop 1 \\
10 & 100 & 45 & + & + & - & - & - & + & loop 1 \\
10 & 1000 & 45 & + & + & - & - & - & + & loop 1 \\
5 & 10 & 45 & + & + & - & - & - & + & loop 1 \\
20 & 10 & 45 & + & + & - & - & - & + & loop 1 \\
10 & 10 & 30 & + & + & - & - & - & + & loop 1 \\
10 & 10 & 60 & + & + & - & - & - & + & loop 1
\end{tabular}

Table 1. Simulation result

\section{Conclusion}

On the basis of analysis to the characteristics of medium voltage distribution network single-phase fault system, then to discuss and analyze transient data wavelet transformed. We construct a new line selection criterion, it is based on wavelet transform amplitude, with comparing the subtracting numerical among each loop coefficient. We select the plus or minus sign. This method makes up for the deficiency of the previous wavelet transform and more accurately distinguish the differences after the signal processing. This method is proved to be accurately and reliable by lots of simulation results and it has also much stronger anti-interference capability to applicable to each grounding method of medium voltage distribution network.

\section{Acknowledgment}

Foundation item: This paper is partially aided by Natural Science Foundation of China (61203370, 61305129), Shandong Province Natural Science Foundation (ZR2013CQ017,ZR2012EEM021), a Project of Shandong Province Higher Educational Science and Technology Program (J13LN17).

\section{References}

[1] Shu Hongchun: Fault Line Selection of Distribution Power System. China Machine Press(2008) [2]Zhao Xiaobin: About power system neutral point of single-phase earth fault analysis and processing. China Science and Technology Information. 9,138-138(2012)

[3]Liu Linfei, Fang shan, Xin Zili: The Study of the Fault Line Selection of the Small Current Grounding System based on Wavelet Analysis. Science \& Technology Vision, 21, 139-140 (2014) [4]Deng Yong, Zhao Lei, Zhang Hongtao: The transient zero sequence current line selection method in the application of small current grounding system, Electrotechnical Application. 11, 34-37 (2012) [5]He Zhengyou: The application of wavelet analysis in power system transient signal processing.China Electric Power Press(2011)

[6]Gaoxiang, LiJunqing: Survey of the methods of Fault Line Selection Based on Small Current 
Grounding System of Distribution Network, Science and Technology, 11, 37 (2014)

[7]JinZhiqing: The Study of the Transient Selection and Disturbance Recognition Based on Wavelet Decomposition and Reconsitutuin, Shanxi Science and Technology, 4, 67-71(2012) 